\title{
Indicadores Emocionais no Desenho da Figura Humana de Crianças Transplantadas de Órgãos
}

\author{
Emotional Indicators in the Draw-a-Person Test of Transplanted Children
}

\author{
Elisa Kern de Castro*,a \& Bernardo Moreno-Jiménez ${ }^{b}$ \\ ${ }^{a}$ Universidade do Vale do Rio dos Sinos \& ${ }^{b}$ Universidade Antônoma de Madri
}

\begin{abstract}
Resumo
O objetivo do estudo foi examinar a presença de indicadores emocionais no Desenho da Figura Humana (DFH), utilizando o sistema Koppitz, em crianças transplantadas e sem problemas de saúde, comparandoas de acordo com o sexo. Quarenta e sete crianças transplantadas e 88 sem problemas de saúde com idades entre 5 e 12 anos completaram o DFH e responderam a uma entrevista sobre o mesmo. O teste $t$ mostrou que os meninos transplantados apresentaram mais indicadores emocionais que os meninos sem problemas de saúde. Análise de regressão logística evidenciou que o indicador integração pobre das partes e DFH na posição esquerda do papel são características típicas das crianças transplantadas. Os resultados indicam que as crianças transplantadas, especialmente os meninos, possuem maiores riscos de apresentar problemas psicológicos. Uma avaliação sistemática desses pacientes é necessária para minimizar eventuais consequiências emocionais relacionadas à doença crônica e ao transplante de órgãos na infância.

Palavras-chave: Infância; Transplante de órgãos; Problemas psicológicos; Desenho da figura humana.
\end{abstract}

\begin{abstract}
This study aims at examining the presence of emotional indicators in the Draw-A- Person Test (DAP), employing the Koppitz Scoring System, in transplanted and healthy children comparing them according to their gender. Forty-seven transplanted and 88 healthy children aged 5-12 completed the DAP and answered an interview about it. The T-test showed that male transplanted children presented more emotional indicators than male healthy children. Logistic regression analysis (LRA) demonstrated that 'poor integration of parts' indicator and DAP in the left side of the paper are typical characteristics of transplanted children. It seems that transplanted children, especially boys, are at higher risk to present psychological problems. A systematic evaluation of those patients is necessary to minimize emotional consequences due to chronic disease and organ transplantation in childhood.

Keywords: Childhood; Organ transplantation; Psychological problems; Draw-a-person test.
\end{abstract}

O transplante de órgãos é um procedimento cirúrgico que visa a tratar doenças terminais irreversíveis de órgãos vitais (Bueno \& Estévez, 2000; Goodwin, Bickerton, Parsons, \& Lask, 2000; Harmon, 1998; Reed, Baz, McGinn, \& Schofield, 2001). Os pacientes com indicação de realização desse procedimento podem se beneficiar de tempo de sobrevivência e melhora na qualidade de vida geral (Lovera et al., 2000; Reed et al., 2001; Streisand \& Tercyak, 2001).
* Endereço para correspondência: Universidade do Vale do Rio dos Sinos, Centro de Ciências da Saúde, Avenida Unisinos, 950, Sala 2B117, Cristo Rei, São Leopoldo, RS, Brasil, CEP 93022-000. E-mail: elisa.kerndecastro@gmail.com

$\mathrm{O}$ artigo é derivado de parte da tese de doutorado da $1^{\mathrm{a}}$ autora, sob supervisão do segundo autor, apresentada no Programa de Doutorado em Psicologia Clínica e da Saúde da Universidade Autônoma de Madri. Os autores agradecem à CAPES pelo auxílio financeiro fornecido através de bolsa de doutorado pleno no exterior concedida à $1^{\mathrm{a}}$ autora (2002-2006)
Crianças doentes estão vulneráveis a estressores como hospitalizações freqüentes e cirurgias (Trianes, 2002). Além disso, as consequiências do transplante para o desenvolvimento emocional da criança ainda não são totalmente conhecidas, e dependem de características da doença, dos recursos psicológicos da criança e de sua família e de seu ambiente social (Castro \& MorenoJiménez, 2004, 2007). Por isso, situações de doença e transplante na idade pediátrica requerem uma rigorosa adesão ao tratamento médico e cuidados com a saúde (Watson, 2000).

A literatura sobre crianças doentes e sua relação com o aparecimento de sintomas psicopatológicos apontam para um maior risco desses pacientes em apresentar desajustes emocionais e comportamentais de diversos tipos (Bradford, 1997; Immelt, 2006). Contudo, outros estudos indicam que uma minoria significativa de crianças com doenças crônicas físicas apresenta de fato problemas psicológicos (Gartstein, Noll, \& Vannatta, 2000; Wallander \& Varni, 1998). Para os autores, os esforços 
que se têm feito para entender as necessidades em saúde mental dessas crianças têm sido problemáticos pelas limitações metodológicas: a maioria dos estudos se fundamenta em informações dos pais sobre o comportamento da criança e pouco se conhece sobre a percepção das próprias crianças. Porém sabe-se que a opinião dos pais tem importante viés, especialmente pelo fato de que muitos progenitores de crianças doentes crônicas apresentam comportamentos superprotetores (Gartstein et al., 2000; Remor, Ulla, Ramos, Arranz, \& Hernández-Navarro, 2003), dispõem de pouco apoio social (Brewer et al., 2007) e tem riscos de apresentarem estresse pós-traumático (Farley et al., 2007; Young et al., 2003).

O uso de instrumentos gráficos, como o desenho da figura humana, pode ser uma técnica alternativa para avaliar o estado psicológico de crianças doentes já que é uma atividade lúdica, divertida, que não requer linguagem verbal e que estimula a criança a participar (DiCarlo, Gibbons, Kaminsky, Wright, \& Stiles, 2003; Matto, 2002; Rae \& Hyland, 2001; Thomas \& Jolley, 1998). De acordo com Koppitz (1966, 1973), distorções no desenho da figura humana são consideradas indicadores de problemas emocionais na criança. A presença de dois ou mais indicadores emocionais da lista de 30 que foi proposta pode sugerir desajuste psicológico. Os dois estudos encontrados que utilizaram o $\mathrm{DFH}^{1}$ com crianças transplantadas se basearam nesse método (Adebäck, Nemeth, \& Fishler, 2003; Gritti et al., 2001). Ambas as pesquisas mostraram que o DFH pode ser bastante útil na hora de identificar pacientes transplantados com problemas emocionais e observaram que os DFH dessas crianças possuem algumas particularidades, como a presença de figura pequena.

Assim, o objetivo do presente estudo é examinar a presença de indicadores emocionais no desenho da figura humana de crianças transplantadas e sem problemas de saúde, comparando-as de acordo com o sexo. Busca-se, além disso, identificar aqueles indicadores emocionais que possam caracterizar a amostra de crianças transplantadas.

\section{Método}

\section{Delineamento}

Estudo ex post facto com um grupo quase-controle (Montero \& León, 2005): crianças transplantadas de órgãos sólidos (rim, fígado e coração) X crianças sem problemas de saúde. Para cada participante e para cada grupo foram examinadas a presença de indicadores emocionais no desenho da figura humana.

\section{Participantes}

Foram avaliadas um total de 135 crianças, das quais 47 eram receptoras de transplante de órgãos sólidos e 88 eram crianças sem problemas de saúde, todas entre 5 e

${ }^{1}$ Será utilizado a abreviação DFH para Desenho da Figura Humana.
12 anos de idade (media 109,88 meses, $D P=23,29$ ). Todos os participantes transplantados de órgãos estavam em tratamento de rotina pós-transplante em três hospitais públicos de Madri, Espanha. Durante o período de três meses em que os dados foram coletados, foram avaliadas todas as crianças transplantadas das equipes de transplante renal e cardíaco, e metade das crianças transplantadas de fígado que preenchiam os critérios para participar do estudo. Os participantes sem problemas de saúde eram estudantes da rede pública de educação de Madri, Espanha. Os critérios de inclusão para participar da pesquisa foram: idade igual ou superior a 5 anos e inferior a 12 anos e 11 meses, estar transplantado (grupo de crianças transplantadas), e aceitação em participar da investigação por parte dos pais ou responsáveis, assinando o termo de Consentimento Informado. Foram excluídas da amostra aquelas crianças transplantadas que apresentavam dano neurológico devido à doença crônica. A Tabela 1 mostra as principais características da amostra:

\section{Procedimentos}

Com os pacientes transplantados de órgãos, a coleta de dados ocorreu nos dias de consultas ambulatoriais das equipes contatadas. Depois da aceitação por parte dos chefes de equipe de transplantes em colaborar com o estudo, a primeira autora passou a estar presente nos dias de consultas externas dos pacientes transplantados. Os procedimentos para a coleta de dados foram sugeridos pelas equipes para que se adaptassem da melhor forma à rotina existente. As crianças e seu responsável passavam toda a manhã em atividades de consultas médicas e exames, e nesses intervalos ocorria a aplicação dos instrumentos, de maneira individual.

Com o grupo de crianças sem problemas de saúde, a coleta de dados ocorreu numa escola pública de Madri, Espanha. Após a aceitação da direção da escola em colaborar com o estudo, foram enviadas 200 cartas a todos os pais ou responsáveis de crianças entre 5 e 12 anos de idade explicando-lhes os objetivos da pesquisa, pedindo-lhes que assinassem o Consentimento Informado e que respondessem às informações sócio-demográficas. 88 famílias aceitaram participar do estudo. A coleta de dados com as crianças ocorreu de maneira coletiva na própria escola. As crianças foram divididas em grupos de acordo com suas idades.

Com respeito aos procedimentos éticos, todos os responsáveis pelas crianças assinaram o Termo de Consentimento Livre e Esclarecido. Nos hospitais em que foi realizada a coleta de dados, o projeto foi aprovado pelos chefes das equipes de transplante já que não havia comitê de ética nos locais. Na escola, a diretora e as professoras das turmas contatadas também aprovaram a realização da pesquisa.

\section{Instrumentos}

Informações Sócio-Demográficas. Recolhe um conjunto de informações relevantes sobre os dados sóciodemográficos da criança e da família (idade da criança, 
Tabela 1

Dados Sócio-Demográficos e Clínicos dos Participantes

\begin{tabular}{|c|c|c|c|c|c|}
\hline & Crianças sps & Crianças tx & tx renal & tx cardíaco & tx hepático \\
\hline Participantes & 88 & 47 & 8 & 10 & 29 \\
\hline \multicolumn{6}{|l|}{ Gênero } \\
\hline Masculino & $45.5 \%$ & $47.9 \%$ & $62.5 \%$ & $60.0 \%$ & $37.9 \%$ \\
\hline Feminino & $54.5 \%$ & $52.1 \%$ & $37.5 \%$ & $40.0 \%$ & $62.1 \%$ \\
\hline \multirow[t]{2}{*}{ Idade (meses) } & 111,50 & 106,85 & 112,25 & 108,70 & 104,72 \\
\hline & $(22,51)$ & $(24,56)$ & $(28,54)$ & $(20,23)$ & $(25,32)$ \\
\hline \multirow[t]{2}{*}{ Idade no diagnóstico (meses) } & - & 14,85 & 25,44 & 20,35 & 10,03 \\
\hline & - & $(29,41)$ & $(34,97)$ & $(37,55)$ & $(24,39)$ \\
\hline \multirow[t]{2}{*}{ Idade no tx (meses) } & - & 36,68 & 66,12 & 32,60 & 29,96 \\
\hline & & $(35,23)$ & $(27,77)$ & $(42,41)$ & $(41,10)$ \\
\hline \multirow[t]{2}{*}{ Tempo em lista de espera (meses) } & - & 2,72 & 7,00 & 2,69 & 1,30 \\
\hline & & $(3,30)$ & $(4,69)$ & $(1,96)$ & $(1,55)$ \\
\hline \multicolumn{6}{|l|}{$N^{o}$ de transplantes } \\
\hline 1 & - & $89,6 \%$ & $87,5 \%$ & $100 \%$ & $86,2 \%$ \\
\hline 2 & - & $6,3 \%$ & $0 \%$ & $0 \%$ & $10,3 \%$ \\
\hline 3 & - & $4,2 \%$ & $12,5 \%$ & $0 \%$ & $3,4 \%$ \\
\hline \multicolumn{6}{|l|}{ Tipo de doador } \\
\hline Cadáver & - & $95,74 \%$ & $87,50 \%$ & $100 \%$ & $96,55 \%$ \\
\hline Vivo (mãe) & - & $4,26 \%$ & $12,50 \%$ & $0 \%$ & $3,45 \%$ \\
\hline \multicolumn{6}{|l|}{ Rejeição ao tx } \\
\hline Não & - & $70,21 \%$ & $75,00 \%$ & $80,00 \%$ & $65,52 \%$ \\
\hline Sim & - & $29,79 \%$ & $25,00 \%$ & $20,00 \%$ & $34,48 \%$ \\
\hline \multicolumn{6}{|l|}{ Estado de saúde } \\
\hline Estável & - & $95,74 \%$ & $87,50 \%$ & $90,00 \%$ & $100 \%$ \\
\hline Em lista p/ novo transplante & - & $4,26 \%$ & $12,50 \%$ & $10,00 \%$ & $0 \%$ \\
\hline \multicolumn{6}{|l|}{ Escolaridade } \\
\hline Esperada & 100 & $85,11 \%$ & $87,50 \%$ & $80,00 \%$ & $86,21 \%$ \\
\hline Atrasada (até 2 anos) & $0 \%$ & $12,76 \%$ & $12,50 \%$ & $10 \%$ & $13,79 \%$ \\
\hline Bastante atrasada & $0 \%$ & $2,13 \%$ & $(0 \%)$ & $10 \%$ & $0 \%$ \\
\hline \multirow[t]{2}{*}{ Idade pai (anos) } & 39,90 & 42,22 & 41,87 & 40,70 & 42,86 \\
\hline & $(4,82)$ & $(5,45)$ & $(5,87)$ & $(5,83)$ & $(5,62)$ \\
\hline \multirow[t]{2}{*}{ Idade mãe (anos) } & 38,08 & 39,06 & 38,75 & 39,00 & 39,18 \\
\hline & $(5,11)$ & $(4,19)$ & $(4,59)$ & $(4,88)$ & $(3,97)$ \\
\hline \multirow[t]{2}{*}{$N^{o}$ de irmãos } & 1,20 & 0,94 & 1,37 & 0,90 & 0,88 \\
\hline & $(0,72)$ & $(0,73)$ & $(1,30)$ & $(0,32)$ & $(0,60)$ \\
\hline
\end{tabular}

Nota. sps $=$ sem problemas de saúde; $\mathrm{tx}=$ transplantado.

profissão dos pais, escolaridade, irmãos) e situações adversas da família (imigração, morte de um dos pais, adoção, etc.).

Informações Clínicas sobre a Doença, Tratamento $e$ Transplante de Órgãos. Coleta informações sobre a doença da criança prévia ao transplante (diagnóstico, idade no diagnóstico, gravidade) e sobre o transplante (idade do paciente no transplante, rejeição, tempo em lista de espera).

Desenho da Figura Humana (Koppitz, 1973). Examina a presença/ausência de indicadores emocionais. Todas as crianças foram solicitadas a desenhar a "uma pessoa com o corpo inteiro", utilizando um lápis e uma folha de papel em branco. Depois do término do desenho, foi realizada uma entrevista sobre a figura humana em que se perguntava às crianças o sexo da figura, idade, o que estava fazendo e como se sentia. Dois juizes treinados avaliaram o DFH e foi calculado o índice kappa baseado em 30\% dos desenhos. O índice encontrado foi de 0,86 , considerado satisfatório.

No presente estudo foram utilizados para análise os 30 indicadores emocionais propostos por Koppitz (1973). A presença de dois ou mais indicadores sugerem problemas emocionais na criança. Essa técnica já foi utilizada em estudos com crianças doentes crônicas (Freire, Cariola, \& Pimentel, 2003; Maldonado, Cariola, Yamada, 
\& Bevilacqua, 2001) e com crianças transplantadas de órgãos (Adebäck et al., 2003; Gritti et al., 2001), mostrando ser útil em identificar problemas emocionais. As respostas à entrevista sobre o DFH foram transcritas e submetidas à análise de conteúdo quantitativa (Laville \& Dione, 1999).

\section{Análise dos Dados}

Inicialmente foi realizada comparação de médias (teste $t$ ) do total de indicadores emocionais dos grupos de crianças transplantadas e sem problemas de saúde, de acordo com o sexo. A seguir foram feitas análises de freqüências e Qui-quadrado de cada um dos indicadores emocionais. Além disso, realizou-se uma análise de regressão logística para verificar se existiam indicadores emocionais que discriminassem aos grupos de crianças transplantadas e sem problemas de saúde.

\section{Resultados}

A Tabela 2 mostra os resultados referentes aos indicadores emocionais e categorias da entrevista. Verificouse que os meninos transplantados demonstraram sinais de pior adaptação emocional nos DFH de maneira evidente em comparação com os meninos sem problemas de saúde. A média de indicadores emocionais dos meninos transplantados foi significativamente mais alta $(t=2,525 ; p<, 01)$ assim como a freqüência de aparecimento de alguns indicadores emocionais específicos, indicando mais problemas psicológicos. Igualmente, os meninos transplantados referiram mais frequentemente que seus desenhos eram de figuras do sexo oposto ao seu, que realizavam pouca atividade e com o papel na posição horizontal. Em contrapartida, os resultados da comparação entre as meninas foram diferentes. A média e o desvio padrão foram muito semelhantes entre os dois grupos, revelando boa adaptação psicológica das meninas transplantadas. Por outro lado, as meninas transplantadas desenharam de maneira mais freqüente o indicador "braços compridos", referiram que suas figuras humanas eram do sexo oposto com idades diferentes às suas, localizadas essencialmente na posição periférica do papel (esquerda ou direita), com traçado de linha de força média ou superior.

A seguir foi realizada uma análise de regressão logística com o objetivo de identificar os indicadores emocionais (30 variáveis categóricas) e categorias da entrevista que poderiam discriminar e caracterizar o grupo de crianças transplantadas. Os coeficientes obtidos permitem verificar a capacidade de cada variável independente em discriminar aos grupos e também a capacidade de prognosticar a classificação dos participantes em cada grupo. A utilização do método de seleção por passos permite incluir no modelo unicamente as variáveis que foram significativas. Para realizar a análise de regressão logística foi empregada como variável de agrupamento o fato de pertencer ao grupo transplantado ou sem problemas de saúde, e como variáveis independentes aqueles indicadores emocionais, categorias da entrevista sobre o DFH e outras categorias que foram significativas na comparação de médias dos grupos de crianças transplantadas e sem problemas de saúde. Portanto, foram incluídas no primeiro bloco de análises os indicadores emocionais integração pobre das partes, assimetria grosseira das extremidades, figura pequena, omissão das mãos, presença de nuvens, chuva ou pássaros voando e omissão dos braços, e no segundo bloco de variáveis atividade que a pessoa está realizando, localização, idade da pessoa desenhada, sexo da pessoa desenhada, presença ou não de solo. A Tabela 3 informa sobre as variáveis incorporadas no modelo em cada um dos passos no bloco 1 , as estimações dos coeficientes, sua significância e o intervalo de confiança. Das seis variáveis independentes incluídas na análise, o método por passos selecionou três: integração pobre das partes, figura pequena e presença de nuvens, chuva ou pássaros voando.

A coluna $\operatorname{Exp}(b)$ indica em que medida a presença de cada um dos indicadores discrimina entre as crianças transplantadas e sem problemas de saúde. Esse índice, que é gerado a partir do quociente entre a probabilidade de que o sujeito seja ou não transplantado em caso de presença ou ausência do indicador, tomaria um valor 1 se o indicador não discriminasse entre os grupos. Por sua vez, quanto mais se aproxime o valor a zero, mais típica será o aparecimento de um indicador concreto nas crianças transplantados, sendo mais típica nas crianças sem problemas de saúde a medida que se distancie do valor de 1 . Como pode observar-se na Tabela 3, os itens integração pobre das partes e figura pequena caracterizam em maior medida as crianças transplantadas, sendo o item figura pequena o que maior poder discriminativo apresenta. A presença de chuva, nuvens ou pássaros voando caracteriza as crianças sem problemas de saúde, tendo um poder discriminativo menor e em sentido inverso aos dois anteriores. Ao incluir-se no modelo as variáveis da entrevista no bloco 2 , observase que no passo 4 apenas o indicador emocional integração pobre das partes permaneceu significativo, mas também foram incluídas as variáveis idade da pessoa desenhada, atividade que está realizando, localização e presença solo. Esses resultados indicam que foi discriminativo para as crianças transplantadas, além da presença do indicador emocional integração pobre das partes, também a localização do desenho do lado esquerdo do papel, enquanto as crianças sem problemas de saúde diferenciaram-se por referir que suas figuras humanas tinham a sua mesma idade e pela presença de solo embaixo de suas figuras.

O resultado da classificação correta das crianças em cada grupo a partir do ponto de corte de 0,5 foi bastante aceitável: $85,6 \%$ em geral, sendo que $86,4 \%$ das crianças transplantadas e $85,2 \%$ das crianças sem problemas de 
Tabela 2

Freqüências, Qui-quadrado e Nível de Significância de cada Indicador Emocional do DFH, Média e Comparação de Médias (teste t) dos Resultados das Crianças Transplantadas de Órgãos e Crianças Sem Problemas de Saúde segundo o Gênero

\begin{tabular}{|c|c|c|c|c|c|c|}
\hline $\begin{array}{l}\text { Indicadores emocionais, localização } \\
\text { e categorias da entrevista }\end{array}$ & $\begin{array}{c}\% \text { de } \\
\text { meninos tx }\end{array}$ & $\begin{array}{c}\% \text { de } \\
\text { meninos sps }\end{array}$ & $\chi^{2}$ & $\begin{array}{l}\% \text { de } \\
\text { meninas tx }\end{array}$ & $\begin{array}{c}\% \text { de } \\
\text { meninas sps }\end{array}$ & $\chi^{2}$ \\
\hline K1. Integração pobre das partes & $22,7 \%$ & $2,5 \%$ & $6,644 * *$ & $8,3 \%$ & $4,2 \%$ & 1,581 \\
\hline$K 2$. Sombreado do rosto & $9,1 \%$ & $5,0 \%$ & 0,394 & $4,2 \%$ & $6,2 \%$ & 0,161 \\
\hline$K 3$. Sombreado do corpo e/ou extremidades & $13,6 \%$ & $10,0 \%$ & 0,187 & $4,2 \%$ & $4,2 \%$ & 0,001 \\
\hline K4. Sombreado de das mãos e/ou pescoço & $4,5 \%$ & $0 \%$ & 1,848 & $0 \%$ & $2,1 \%$ & 0,528 \\
\hline K5. Assimetria grosseira das extremidades & $40,9 \%$ & $15,0 \%$ & $5,195 *$ & $8,3 \%$ & $4,2 \%$ & 0,466 \\
\hline K6. Figura inclinadas & $4,5 \%$ & $0 \%$ & 1,848 & $4,2 \%$ & $0 \%$ & 0,528 \\
\hline$K 7$. Figura pequena & $31,8 \%$ & $0 \%$ & $14,347 * *$ & $12,5 \%$ & $2,1 \%$ & 3,121 \\
\hline K8. Figura grande & $4,5 \%$ & $7,5 \%$ & 0,205 & $0 \%$ & $10,4 \%$ & 0,897 \\
\hline K9. Transparências & $4,5 \%$ & $2,5 \%$ & 0,190 & $4,2 \%$ & $0 \%$ & 1,947 \\
\hline K10. Cabeça pequena & $0 \%$ & $7,5 \%$ & 1,734 & $0 \%$ & $0 \%$ & - \\
\hline K11. Olhos vesgos & $0 \%$ & $15,0 \%$ & $3,654 *$ & $4,2 \%$ & $4,2 \%$ & 0,001 \\
\hline K12. Dentes & $4,5 \%$ & $17,5 \%$ & 2,120 & $4,2 \%$ & $4,2 \%$ & 0,001 \\
\hline K13. Braços curtos & $9,1 \%$ & $7,5 \%$ & 0,048 & $20,8 \%$ & $14,6 \%$ & 0,351 \\
\hline K14. Braços compridos & $9,1 \%$ & $2,5 \%$ & 1,339 & $8,3 \%$ & $0 \%$ & $3,948 *$ \\
\hline K15. Braços pegados ao corpo & $4,5 \%$ & $7,5 \%$ & 0,205 & $12,5 \%$ & $14,6 \%$ & 0,093 \\
\hline K16. Mãos grandes & $13,6 \%$ & $5,0 \%$ & 1,428 & $4,2 \%$ & $0 \%$ & 1,947 \\
\hline K17. Mãos omitidas & $13,6 \%$ & $0 \%$ & $5,732 * *$ & $8,3 \%$ & $4,2 \%$ & 0,466 \\
\hline K18. Pernas juntas & $13,6 \%$ & $7,5 \%$ & 0,611 & $20,8 \%$ & $12,5 \%$ & 0,723 \\
\hline K19. Genitais & $0 \%$ & $5,0 \%$ & 1,137 & $0 \%$ & $0 \%$ & - \\
\hline$K 20$. Monstro ou figura grotesca & $4,5 \%$ & $0 \%$ & 1,848 & $0 \%$ & $0 \%$ & - \\
\hline K21. Desenho espontâneo de três ou mais figuras & $9,1 \%$ & $7,5 \%$ & 0,048 & $4,2 \%$ & $4,2 \%$ & 0,001 \\
\hline K22. Nuvens, chuva, neve ou pássaros voando & $0 \%$ & $20,0 \%$ & $5,052 *$ & $16,0 \%$ & $29,2 \%$ & 1,534 \\
\hline K23. Omissão dos olhos & $4,5 \%$ & $0 \%$ & 1,848 & $0 \%$ & $0 \%$ & - \\
\hline K24. Omissão do nariz & $22,3 \%$ & $22,5 \%$ & 0,176 & $8,3 \%$ & $12,5 \%$ & 0,341 \\
\hline K25. Omissão da boca & $0 \%$ & $0 \%$ & - & $0 \%$ & $0 \%$ & - \\
\hline K26. Omissão do corpo & $0 \%$ & $0 \%$ & - & $0 \%$ & $0 \%$ & - \\
\hline K27. Omissão dos braços & $9,1 \%$ & $0 \%$ & $3,758 *$ & $0 \%$ & $0 \%$ & - \\
\hline K28. Omissão das pernas & $4,5 \%$ & $0 \%$ & 1,848 & $0 \%$ & $0 \%$ & - \\
\hline K29. Omissão dos pés & $0 \%$ & $0 \%$ & - & $0 \%$ & $0 \%$ & - \\
\hline K30. Omissão do pescoço & $36,4 \%$ & $20,0 \%$ & 1,985 & $12,5 \%$ & $22,9 \%$ & 0,482 \\
\hline \multicolumn{7}{|l|}{ Sexo da pessoa desenhada } \\
\hline Mesmo sexo & $71,4 \%$ & $85,0 \%$ & \multirow{3}{*}{$8,250 * *$} & $70,8 \%$ & $85,4 \%$ & \multirow[t]{3}{*}{$6,148 *$} \\
\hline Sexo oposto & $28,6 \%$ & $5,0 \%$ & & $29,2 \%$ & $8,3 \%$ & \\
\hline Ambos sexos (2 pessoas ou mais) & $0 \%$ & $10,0 \%$ & & $0 \%$ & $6,2 \%$ & \\
\hline \multicolumn{7}{|l|}{ Idade da pessoa desenhada } \\
\hline Idade aproximada à da criança & $38,1 \%$ & $59,0 \%$ & \multirow{3}{*}{2,408} & $54,2 \%$ & $77,1 \%$ & \multirow[t]{3}{*}{$8,892 * *$} \\
\hline Mais jovem & $4,8 \%$ & $2,5 \%$ & & $16,7 \%$ & $0 \%$ & \\
\hline Mais velho & $57,1 \%$ & $38,5 \%$ & & $29,2 \%$ & $22,9 \%$ & \\
\hline \multicolumn{7}{|l|}{ Atividade da pessoa desenhada } \\
\hline Está parada/não está fazendo nada/é uma foto & $47,6 \%$ & $11,4 \%$ & \multirow{5}{*}{$15,452 * *$} & $33,3 \%$ & $14,6 \%$ & \multirow{5}{*}{3,823} \\
\hline Está olhando/pensando & $4,7 \%$ & $14,3 \%$ & & $8,325,0 \%$ & $16,7 \%$ & \\
\hline Está cumprimentando/passeando & $9,5 \%$ & $28,6 \%$ & & $25,0 \%$ & $18,7 \%$ & \\
\hline Está brincando & $28,6 \%$ & $31,5 \%$ & & $29,2 \%$ & $45,8 \%$ & \\
\hline Outros & $9,5 \%$ & $14,3 \%$ & & $4,1 \%$ & $4,2 \%$ & \\
\hline \multicolumn{7}{|l|}{ Sentimentos da pessoa desenhada } \\
\hline Sentimentos positivos (feliz, contente, etc.) & $90,5 \%$ & $87,5 \%$ & \multirow{4}{*}{3,496} & $87,5 \%$ & 87,5 & \multirow{4}{*}{0,375} \\
\hline Sentimentos negativos (triste, preocupado, etc.) & $4,8 \%$ & $5,0 \%$ & & $0 \%$ & $4,2 \%$ & \\
\hline Sentimentos positivos e negativos & $0 \%$ & $0 \%$ & & $4,1 \%$ & $2,1 \%$ & \\
\hline Não sabe/não respondeu & $4,8 \%$ & $07,5 \%$ & & $8,3 \%$ & $6,2 \%$ & \\
\hline Desenho realizado com o papel na posição vertical & $l \quad 40,9 \%$ & $80,0 \%$ & $8,621 * *$ & $33,3 \%$ & $58,3 \%$ & 3,280 \\
\hline Desenho realizado com o papel na posição horizontc & tal $59,1 \%$ & $20,0 \%$ & & 66,6 & $41,7 \%$ & \\
\hline
\end{tabular}


Castro, E. K. \& Moreno-Jiménez, B. (2010). Indicadores Emocionais no Desenho da Figura Humana de Crianças Transplantadas de Órgãos.

\begin{tabular}{lrrrrrr} 
Localização do desenho no papel 1 & & & & & & \\
$\quad$ Quadrante superior & $27,3 \%$ & $20,0 \%$ & 0,646 & $16,7 \%$ & $10,4 \%$ & 2,227 \\
$\quad$ Quadrante inferior & $22,7 \%$ & $30,0 \%$ & & $45,8 \%$ & $31,2 \%$ & \\
$\quad$ Central & $50,0 \%$ & $50,0 \%$ & & $37,5 \%$ & $58,3 \%$ & \\
Localização do desenho no papel & & & & & & \\
$\quad$ Esquerda & $40,9 \%$ & $42,5 \%$ & 0,495 & $66,7 \%$ & $37,5 \%$ & $8,112^{* *}$ \\
$\quad$ Direita & $9,1 \%$ & $5,0 \%$ & & $12,5 \%$ & $4,2 \%$ & \\
$\quad$ Centro & $50,0 \%$ & $52,5 \%$ & & $20,8 \%$ & $58,3 \%$ & \\
$\quad$ Traçado da linha & & & & & & \\
$\quad$ Pouca força & $9,1 \%$ & $10,0 \%$ & 0,291 & $0 \%$ & $21,9 \%$ & $6,858^{*}$ \\
$\quad$ Media força & $68,2 \%$ & $60,0 \%$ & & $75,0 \%$ & $58,3 \%$ & \\
$\quad$ Muita força & $22,7 \%$ & $30,0 \%$ & & $25,0 \%$ & $18,7 \%$ & \\
$\quad$ & & & $t$ test & & & $t$ test \\
Media dos Indicadores Emocionais (total) & 3,00 & 1,83 & $2,525 * *$ & 1,63 & 1,61 & 0,049 \\
& $(2,37)$ & $(1,32)$ & & $(1,36)$ & $(1,33)$ & \\
\hline
\end{tabular}

Nota. ${ }^{*} p<.05 ; * *<<.01 ; \mathrm{sps}=$ sem problemas de saúde; $\mathrm{tx}=$ transplantado.

Tabela 3

Variáveis Incluídas no Modelo (estimações e nível de significância dos coeficientes)

Modelo 1 (indicadores emocionais que foram significativos)

\begin{tabular}{llrrrrr} 
& & \multicolumn{4}{c}{ I.C. $95 \%$ para $\operatorname{Exp}(\beta)$} \\
& & $(\beta)$ & sig & $\operatorname{Exp}(\beta)$ & Inferior & Superior \\
\hline Passo 3 & K1 integração pobre das partes & $-1,89$ & 0,02 & 0,15 & 0,03 & 0,71 \\
& K7 figura pequena & $-3,08$ & 0,00 & 0,05 & 0,01 & 0,42 \\
& K22 nuvens, chuva, pássaros & 1,73 & 0,02 & 0,62 & 1,36 & 23,27
\end{tabular}

Modelo 2 (indicadores emocionais e categorias da entrevista que foram significativos)

\begin{tabular}{llrrrrr} 
& \multicolumn{5}{c}{ I.C. $95 \%$ para $\operatorname{Exp}(\beta)$} \\
& $(\beta)$ & sig & $\operatorname{Exp}(\beta)$ & Inferior & Superior \\
\hline Passo 4 & K1 integração pobre das partes & $-2,81$ & 0,03 & 0,06 & 0,01 & 0,72 \\
& Idade (DFH com idade semelhante à da criança) & 1,94 & 0,01 & 6,97 & 1,49 & 32,60 \\
& Localização (DFH lado esquerdo) & $-3,63$ & 0,00 & 0,03 & 0,00 & 0,16 \\
& Presença de solo & 4,70 & 0,00 & 109,85 & 9,21 & 1310,17 \\
\hline
\end{tabular}

saúde foram classificadas corretamente. Esse resultado revela que o modelo que incluiu indicadores emocionais e categorias da entrevista sobre o DFH foi válido para predizer o pertencimento das crianças tanto transplantadas como sem problemas de saúde em seu grupo.

\section{Discussão}

A finalidade do presente estudo foi avaliar os indicadores emocionais presentes no Desenho da Figura Humana de crianças transplantadas de órgãos e crianças sem problemas de saúde segundo o sexo, identificando possíveis indicadores que caracterizassem o desenho das crianças transplantadas. A pesquisa foi realizada com um caráter eminentemente exploratório. Apesar de que existem na literatura evidências empíricas sobre os efeitos psicológicos do transplante pediátrico (Adebäck et al., 2003; Castro, Moreno-Jimenez, \& Rodríguez-Carvajal, 2007; Gritti et al., 2001; Gritti et al., 2006), a informação não está suficientemente sistematizada e não abarca todos os aspectos examinados e o instrumento utilizado aqui.

Entre os resultados descritos que se referem à expressão da própria criança sobre seu estado psicológico, destacam-se algumas particularidades entre os grupos de crianças transplantadas e sem problemas de saúde que parecem estar mostrando a influência negativa da história de doença e transplante nas suas vidas. Em geral, as crianças transplantadas apresentaram uma média de indicadores emocionais superior às crianças sem problemas de saúde. No entanto, somente para os meninos transplantados essa média foi significativamente superior à média dos meninos sem problemas de saúde. Segundo Arteche (2006), o DFH como instrumento de avaliação de indicadores emocionais é melhor preditor de problemas de externalização do que de internalização. Portanto, é possível que a técnica utilizada tenha sido mais sensível ao tipo de problemática apresentada pelos meninos, já que frente a estressores eles costumam 
apresentar esse tipo de problemas de comportamento do que as meninas.

Os indicadores emocionais que apareceram com mais freqüência nos desenhos dos meninos transplantados de forma significativa foram: integração pobre das partes, assimetria grosseira das extremidades, figura pequena, omissão das mãos. Apesar de que não existe uma relação inequívoca entre um indicador isolado do desenho da figura humana e uma característica determinada de personalidade ou de comportamento e não seja adequando realizar um diagnóstico de dificuldades das crianças baseado somente nesses indicadores, Koppitz (1973) salienta que eles costumam aparecer mais frequentemente nos desenhos de crianças com algum tipo de problemática. A investigação de Gritti et al. (2001) também encontrou que crianças transplantadas de fígado desenharam mais frequentemente figuras humanas pequenas.

Ainda segundo Koppitz (1973), para que um indicador emocional seja válido este não deve aparecer com freqüência superior a $16 \%$ na população clínica. Ao observarmos a Tabela 2 , verifica-se que para os meninos o indicador "omissão do nariz" aparece com freqüência superior a $16 \%$ tanto no grupo de crianças transplantadas como no grupo de crianças sem problemas de saúde, assim como o indicador "Nuvens, chuva, neve ou pássaros voando" para as meninas. Segundo esse critério, esses indicadores não seriam válidos para examinar a presença de problemas emocionais nas crianças avaliadas. No entanto, futuros estudos deverão centrar-se na adequação de alguns desses indicadores emocionais para o exame de problemas psicológicos nas crianças.

Os resultados da análise de regressão logística mostraram que o indicador emocional integração pobre das partes e a localização do DFH no lado esquerdo do papel caracterizaram as crianças transplantadas de maneira significativa, enquanto a presença de solo e o fato de que as figuras humanas tinham o mesmo sexo da criança que a desenhou caracterizaram as crianças sem problemas de saúde. Esses resultados sugerem que a experiência da doença crônica e do transplante de órgãos afeta o estado psicológico de muitas crianças devido ao elevado número de indicadores emocionais encontrados, e que essas crianças são capazes de expressar suas percepções e temores através do DFH. Koppitz (1973) afirmou que crianças com incapacidades ou doentes refletem suas preocupações com o corpo nas suas figuras humanas, apesar de que a preocupação pelo seu problema de saúde possa aumentar ou diminuir na medida em que vão crescendo, e sua representação no desenho pode sofrer mudanças. Rae (1991) foi além e referiu que crianças doentes e/ou hospitalizadas costumam desenhar figuras humanas indo ao médico (representação de sua experiência), recebendo uma injeção (representação do seu medo) ou ganhando um brinquedo (representação do seu desejo). No presente estudo nenhuma criança transplantada desenhou figuras humanas doentes, numa cama ou no hospital.
Os resultados da análise da entrevista sobre o DFH mostraram que as crianças transplantadas desenharam mais frequentemente que as crianças sem problemas de saúde a pessoas do sexo oposto. Os desenhos dos meninos transplantados também se caracterizaram pela tarefa pouco ativa que estava sendo realizada pela figura humana e por utilizar o papel mais frequentemente na posição horizontal que os meninos sem problemas de saúde. Em contrapartida, as meninas transplantadas desenharam de maneira mais freqüente a figuras humanas mais velhas ou mais jovens que a idade delas próprias e na posição periférica do papel em comparação com as crianças sem problemas de saúde. Segundo Koppitz (1973), a pessoa a quem a criança desenha em geral é ela mesma, e, portanto tem o mesmo sexo e idade aproximada à sua. Não obstante, se a criança desenha a outra pessoa isso não necessariamente indicaria um problema, pois poderia também estar revelando uma preocupação positiva com relação a essa pessoa. Com base nessas hipóteses formuladas pela autora, é possível pensar que parte das crianças transplantadas não desenhou a si mesma já que suas figuras mais frequentemente eram do sexo oposto e tinham idades diferentes das suas. É provável que seja muito doloroso para as crianças transplantadas desenhar a si mesmas já que seus corpos foram muito manipulados e levam dentro 'algo' que era de outra pessoa. De qualquer maneira, não foram encontrados estudos que tenham utilizado entrevista semelhante sobre o DFH e, portanto não existem evidências empíricas suficientes que apóiem as hipóteses levantas. Na prática clínica, talvez fosse possível esclarecer essas questões considerando a individualidade e a história de cada paciente, mas a partir dos dados coletados na presente pesquisa não é possível chegar a conclusões definitivas sobre o tema.

Com relação às atividades que desempenhavam as figuras humanas, a análise de conteúdo das respostas das crianças demonstrou que os DFH das crianças transplantadas mais frequentemente não faziam nada, estavam numa foto ou pensando, ou seja, estavam em atitudes indicativas de passividade, enquanto as figuras das crianças sem problemas de saúde estavam em atividade, brincando ou passeando. Esse resultado pode estar refletindo as limitações da vida cotidiana das crianças transplantadas, pois podem ter dificuldades para brincar ou passear como gostariam, e que existe certa apatia na hora de desenvolver atividades.

Com relação à posição periférica dos DFH, Koppitz não trata do tema. Entretanto, Hammer (1969), um dos autores clássicos e pioneiros na utilização dos desenhos, dizia que quando aparecem desenhos descentrados temos que levantar a hipótese da presença de características dependentes e incontroladas da pessoa. Por não haver estudos controlados sobre o tema, essas hipóteses sugeridas pelo autor devem ser interpretadas com muita cautela. 


\section{Considerações Finais}

Os resultados desse estudo apontam que o transplante de órgãos pode afetar emocionalmente as crianças, resultado obtido a partir da sua própria expressão no desenho da figura humana. No entanto, são necessários mais estudos para entender que variáveis da doença e do transplante podem afetar de maneira mais evidente as crianças.

É importante destacar que apesar de que não se pode falar de uma relação direta entre transplante pediátrico e funcionamento psicológico do paciente, devemos estar conscientes que a doença crônica e o transplante são acontecimentos que tem efeitos no desenvolvimento infantil. Os cuidados diários com a saúde, as visitas freqüentes ao médico, a ingestão de remédios, o medo a uma futura rejeição do órgão e o fato de estar consciente de ter dentro do próprio corpo um órgão que era de outra pessoa são fatos que limitam a vida e o desenvolvimento da criança transplantada e que a família e os profissionais de saúde têm que ter presentes para poder ajudá-los.

A seleção dos participantes ocorreu a partir do contato com quatro equipes de transplantes de três hospitais diferentes. O interesse por parte dos pacientes e de seus responsáveis em participar da pesquisa foi excelente, pois todas as famílias contatadas aceitaram participar do estudo. Além disso, muitos pais se mostraram interessados em conhecer os resultados da investigação.

Cabe comentar que o presente trabalho foi realizado com crianças transplantas de rim, fígado e coração, e que não se observaram particularidades na adaptação psicológica entre os três subgrupos de pacientes. Poucas pesquisas até o momento utilizaram amostras de pacientes pediátricos transplantados de diferentes órgãos, e não foi possível até o momento verificar a influência do tipo de órgão transplantado para o estado emocional da criança. Talvez as diferenças não tenham aparecido ainda porque as amostras estudadas em geral são pequenas, as idades dos participantes são muito variadas e os instrumentos utilizados não são suficientemente sensíveis para captar esses nuances nessa população.

É importante, também, que futuros estudos possam utilizar diferentes instrumentos de avaliação psicológica das crianças transplantadas que possam trazer dados complementares sobre o funcionamento psicológico desses pacientes. Por exemplo, a utilização de técnicas projetivas, instrumentos psicométricos e entrevistas com os pais podem fornecer informações importantes não só dos possíveis problemas psicológicos das crianças transplantadas, mas também do funcionamento familiar onde elas estão inseridas.

Devido a que a avaliação das crianças transplantadas foi realizada na ausência de qualquer programa de apoio psicológico de rotina junto às equipes de transplante contatadas, os dados sugerem fortemente a necessidade de intervenção psicológica com pacientes pediátricos transplantados. Seria recomendável que a intervenção clínica começasse já no período do diagnóstico da doença crônica e antes do transplante, proporcionando assim um maior apoio ao paciente e à sua família para lidar com a situação, prevenindo futuros problemas psicológicos decorrentes dessa experiência. Esse estudo adverte para o risco de problemas psicológicos de crianças transplantadas quando não há programas de apoio psicológico específicos para essa população.

\section{Referências}

Adebäck, P., Nemeth, A., \& Fishler, B. (2003). Cognitive and emotional outcome after pediatric liver transplantion. Pediatric Transplantation, 7(5), 385-389.

Arteche, A. (2006). Construção de uma escala de avaliação dos indicadores emocionais do DFH. Tese de Doutorado não-publicada, Universidade Federal do Rio Grande do Sul, Porto Alegre, RS

Bradford, R. (1997). Children, families and chronic disease. London: Routledge.

Brewer, H. M., Smith, J. A., Eatough, V., Stanley, C. A., Glendinning, N. W., \& Quarrell, O. W. (2007). Caring for a child with juvenile Huntingon's disease: Helpful and unhelpful support. Child Health Care, 11(1), 40-52.

Bueno, J., \& Estévez, J. V. (2000). Futuro del trasplante en el siglo XXI. Revista Española de Pediatría, 56, 119-126.

Castro, E. K., \& Moreno-Jiménez, B. (2004). O transplante de órgãos pediátrico: Papel do psicólogo infantil. Pediatria Moderna, 40(6), 266-269.

Castro, E. K., \& Moreno-Jiménez, B. (2007). Resiliencia en niños enfermos crónicos: aspectos teóricos. Psicología em Estudo, 12(1), 81-86.

Castro, E. K., Moreno-Jiménez, B., \& Rodríguez-Carvajal, R. (2007). Psychological well-being in adults transplanted in childhood. Pediatric Transplantation, 11, 272-278.

DiCarlo, M. A., Gibbons, J. L., Kaminsky, D. C., Wright, J. D., \& Stiles, D. A. (2003). Street children's drawings: Windows into their life circumstances and aspirations. International Social Work, 43(1), 107-120.

Farley, L. M., DeMaso, D. R., D’Angelo, E., Kinnamon, C., Bastardi, H., Hill, C. E., et al. (2007). Parenting stress and parental post-traumatic stress disorder in families after pediatric heart transplantation. Journal of Heart and Lung Transplantation, 26(2), 120-126.

Freire, L. S., Cariola, T. C., \& Pimentel, M. C. (2003). O desenho da figura humana em pré-adolescentes portadores de fissura lábio-palatal, na faixa etária dos 12 a 14 anos. Pediatria Moderna, 39, 93-98.

Gartstein, M. A., Noll, R. B., \& Vannatta, K. (2000). Childhood aggression and chronic illness: Possible protective mechanisms. Journal of Applied Developmental Psychology, 21(3), 315-333.

Goodwin, M., Bickerton, A. M., Parsons, R., \& Lask, B. (2000). Paediatric heart/heart-lung transplantation: A systemic perspective on assessment and preparation. International Journal of Psychiatry in Clinical Practice, 4(2), 93-99.

Gritti, A., Di Sarno, A. M., Comito, M., de Vincenzo, A., de Paola, P., \& Vajro, P. (2001). Psychological impact of liver transplantation on children's inner worlds. Pediatric Transplantation, 5, 37-43. 
Gritti, A., Sicca, F., Di Sarno, A., Cosmo, N., Vajro, S., \& Vajro, P. (2006). Emocional and behavioral problems alter pediatric liver transplantation: A quantitative assessment. Pediatric Transplantation, 10, 205-209.

Hammer, E. F. (1969). Tests proyectivos gráficos. Buenos Aires, Argentina: Paidós.

Harmon, W. (1998). Pediatric organ transplantation. Transplantation Proceedings, 30(5), 1952-1955.

Immelt, S. (2006). Psychological adjustment in young children with chronic medical conditions. Journal of Pediatric Nursing, 21(5), 362-377.

Koppitz, E. M. (1966). Emotional indicators on human figure drawings of children: A validation study. Journal of Clinical Psychology, 22(3), 313-315.

Koppitz, E. M. (1973). El dibujo de la figura humana en los niños: evaluación psicológica (M. J. García \& M. R. Braile, Trans.). Buenos Aires, Argentina: Guadalupe. (Original work published 1968)

Laville, C., \& Dionne, J. (1999). A construção do saber: Manual de metodologia da pesquisa em ciências humanas (L. M. Siman, Trad.). Porto Alegre, RS: Artes Médicas.

Lovera, G., Bertolotti, M., Comazzi, A. M., Clerici, C. A., Gandione, M., Mazzoldi, M., et al. (2000). L'assistenza psicologica nei trapianti d'organo. Annalli dell Istituto Superiori di Sanitá, 36(2), 225-246.

Maldonado, F. P., Cariola, T. C., Yamada, M. O., \& Bevilacqua, M. C. (2001). O desenho da figura humana em crianças com implante coclear e escolaridade. Pediatra Moderna, 38(5), 181-187.

Matto, H. C. (2002). Investigating the validity of the Draw-aperson: Screening procedure for emotional disturbance: A measurement validation study with high-risk youth. Psychological Assessment, 14(2), 221-225.

Montero, I., \& León, O. G. (2005). Sistema de clasificación del método en los informes de investigación en Psicología. International Journal of Clinical and Health Psychology, 5(1), 115-127.

Rae, W. A. (1991). Analyzing drawings of children who are physically ill and hospitalized, using the ipsative method. Child Health Care, 20, 198-207.

Rae, G., \& Hyland, P. (2001). Generalisability and classical test theory analyses of Koppitz's Scoring System for human figure drawings. British Journal of Educational Psychology, 71(3), 369-382.

Reed, A., Baz, M. A., McGinn, L., \& Schofield, R. S. (2001). Solid organ transplantation. In J. R. Rodrigue, Byopsychosocial perspectives on transplantation (pp. 1-18). New York: Kluwer Academic.

Remor, E., Ulla, S., Ramos, J. L., Arranz, P., \& HernándezNavarro, F. (2003). La sobreprotección como un factor de riesgo en la reducción de la autoestima en niños con hemofilia. Psiquis, 24(4), 37-42.

Streisand, R. M., \& Tercyak, K. P. (2001). Evaluating the pediatric transplant patient: General considerations. In J. R. Rodrigue, Byopsychosocial perspectives on transplantation (pp. 71-92). New York: Kluwer Academic.

Thomas, G. V., \& Jolley, R. P. (1998). Drawing conclusions: A re-examination of empirical and conceptual bases for psychological evaluation of children from their drawings. British Journal of Clinical Psychology, 37, 127-139.
Trianes, M. V. (2002). Estrés en la infancia: su prevención y tratamiento. Madrid, España: Narcea.

Wallander, J. L., \& Varni, J. W. (1998, January). Effects of pediatric chronic physical disorders on child and family adjustment. Journal of Child Psychology \& Psychiatry, 39(1), 29-46.

Watson, A. R. (2000). Non-compliance and transfer from paediatric to adult transplant unit. Pediatric Nephrology, 14(6), 469-472.

Young, G. S., Mintzer, L. L., Seacord, D., Castaneda, M., Mesrkhani, V., \& Stuber, M. L. (2003). Symptons of posttraumatic stress disorder in parents of transplant recipients: Incidence, severity, and related factors. Pediatrics, 111(6), 725-731.
Recebido: 09/07/2007

$1^{a}$ revisão: $31 / 03 / 2008$

$2^{a}$ revisão: $20 / 08 / 2008$ Aceite final: $28 / 08 / 2008$ 